\title{
(6) OPEN ACCESS \\ Association between maternal health literacy and child vaccination in India: a cross-sectional study
}

\author{
Mira Johri, ${ }^{1,2}$ S V Subramanian, ${ }^{3}$ Marie-Pierre Sylvestre, ${ }^{1,4}$ Sakshi Dudeja, ${ }^{5}$ \\ Dinesh Chandra, ${ }^{5}$ Georges K Koné, ${ }^{1,6}$ Jitendar K Sharma, ${ }^{7}$ Smriti Pahwa ${ }^{5}$
}

- Additional material is published online only. To view please visit the journal online (http://dx.doi.org/10.1136/jech2014-205436).

For numbered affiliations see end of article.

\section{Correspondence to}

Dr Mira Johri, Unité de Santé Internationale, Centre de Recherche du Centre Hospitalier de I'Université de Montréal (CRCHUM), Tour Saint-Antoine, Porte S03-458, 850 , rue St-Denis, Montréal (Québec), Canada H2X OA9; mira.johri@umontreal.ca

Received 29 December 2014 Revised 3 March 2015 Accepted 10 March 2015 Published Online First 31 March 2015

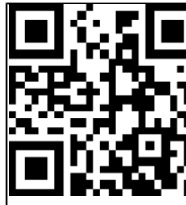

Open Access Scan to access mo free content

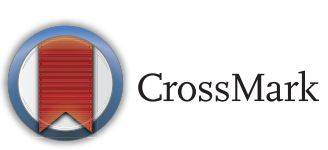

To cite: Johri $\mathrm{M}$ Subramanian SV Sylvestre M-P, et al. $J$ Epidemiol Community Health 2015;69:849-857.

\section{ABSTRACT}

Background Education of mothers may improve child health. We investigated whether maternal health literacy, a rapidly modifiable factor related to mother's education, was associated with children's receipt of vaccines in two underserved Indian communities.

Methods Cross-sectional surveys in an urban and a rural site. We assessed health literacy using Indian child health promotion materials. The outcome was receipt of three doses of diphtheria-tetanus-pertussis (DTP3) vaccine. We used multivariate logistic regression to investigate the relationship between maternal health literacy and vaccination status independently in each site. For both sites, adjusted models considered maternal age, maternal and paternal education, child sex, birth order, household religion and wealth quintile. Rural analyses used multilevel models adjusted for service delivery characteristics. Urban analyses represented cluster characteristics through fixed effects.

Results The rural analysis included 1170 women from 60 villages. The urban analysis included 670 women from nine slum clusters. In each site, crude and adjusted models revealed a positive association between maternal health literacy and DTP3. In the rural site, the adjusted OR was $1.57(95 \% \mathrm{Cl} 1.11$ to $2.21, \mathrm{p}=0.010)$ for those with medium health literacy, and $\mathrm{OR}=1.30(95 \% \mathrm{Cl}$ 0.89 to $1.91, p=0.172$ ) for those with high health literacy. In the urban site, the adjusted OR was 1.10 ( $95 \% \mathrm{Cl} 0.65$ to $1.88, p=0.705$ ) for those with medium health literacy, and $\mathrm{OR}=2.06(95 \% \mathrm{Cl} 1.06$ to 3.99 , $\mathrm{p}=0.032$ ) for those with high health literacy.

Conclusions In these study settings, maternal health literacy is independently associated with child vaccination. Initiatives targeting health literacy could improve vaccination coverage.

\section{INTRODUCTION}

Three decades of research demonstrate a strong, positive link between parental-particularly maternal-education and child health and survival. ${ }^{1}$ A similar relationship can be detected in virtually all countries despite great diversity in living standards, schooling and health systems. ${ }^{2}$

While the relationship between maternal education and child health is effectively universal, debate persists concerning pathways of influence. Focusing on low-income and middle-income countries (LMICs), several mechanisms have been suggested. ${ }^{13}$ These include: (1) changes in reproductive health patterns such as delayed age at child birth, having fewer children and improved birth spacing; (2) better socioeconomic status and living conditions; and (3) behavioural pathways such as greater maternal autonomy, improvements in health beliefs and domestic practices, and greater use of preventive and curative health services. Standard household surveys measure educational achievement but not health knowledge, behaviours or beliefs, limiting their ability to elucidate pathways.

In high-income countries, complementary work on the relationship of education to health has recently invoked the concept of health literacy, often defined as "the degree to which individuals have the capacity to obtain, process and understand basic health information and services needed to make basic health decisions." ${ }^{4}$ Poor health literacy is thought to affect recognition and understanding of health problems and interactions with the healthcare system, ${ }^{5}$ although debate exists on whether health literacy is distinct from general cognitive ability. ${ }^{6}$ Increasingly viewed as a possible determinant of population health in high-income settings, ${ }^{7}$ health literacy has been little studied in LMICs.

We designed a cross-sectional survey to assess whether the well-documented impact of maternal education on child health is correlated with mother's knowledge and understanding. Our objective was to test the hypothesis that maternal health literacy is positively associated with children's receipt of vaccines after adjustment for potential confounding factors, in two communities in India with poor living conditions and health indicators. We chose vaccination as our study end point as it is critical for child survival and flourishing, widely studied, and measurable. Although education is a structural health determinant, health literacy may be rapidly modifiable. If supported, the study hypothesis would suggest a novel strategy to circumvent barriers due to low education and improve child health in LMIC populations with lagging health indicators.

\section{METHODS}

\section{Study design and setting}

We conducted interviewer-administered crosssectional surveys in two sites in India using identical instruments and procedures. Data were collected from a rural district (Hardoi) in the state of Uttar Pradesh from 14 May to 13 July 2013, and from a New Delhi urban slum (Kirti Nagar) from 4 May to 24 October 2013. With a population of four million, ${ }^{8}$ Hardoi figures among 81 (of 640) districts accounting for $1 / 3$ of India's 2012 child mortality, ${ }^{9}$ and receives development funds targeted to India's most backward districts. ${ }^{10}$ Kirti Nagar is an informal settlement of approximately 20000 households living in nine neighbourhoods. Adults are largely migrants from rural India (see web appendix table S1). 
Ethics approval was obtained from the Pratham (India) and CRCHUM (Canada) research ethics committees. All participants gave informed consent before taking part.

\section{Participants and sampling}

All women who lived in a study site and were mothers of a child aged 12-23 months were eligible to participate. Surveyors were instructed to exclude women not able to understand and speak Hindi or Urdu, or cognitively impaired. One woman was excluded from the study for linguistic reasons.

The sampling unit was the household. We sampled one mother per household, and one child per mother, the youngest child in the age group 12-23 months. The rural site employed two-stage probability-proportional-to-size cluster sampling. ${ }^{11} 12$ For the urban site, we conducted a census (see web appendix: sampling).

\section{Variables, data sources and measurement}

All data analysed are from the household surveys. We used identical data collection procedures for both sites and all respondents.

\section{Main outcome}

The main outcome variable is receipt of three dose of diphtheria-tetanus-pertussis (DTP3s) vaccine among children 12-23 months of age, a standard measure of routine immunisation system performance. ${ }^{13}$ We analysed DTP3 as ' 1 ' if the child received three doses of DTP vaccine, and ' 0 ' if the child received zero, one or two doses of DTP vaccine. ${ }^{13}$ Where a child's vaccination card was available, surveyors transcribed data from the card; otherwise, vaccination status was assessed by maternal recall. ${ }^{14-17}$

\section{Exposure variable}

There is no widely accepted tool to measure health literacy. Available instruments were developed in high-income settings and suffer from important scientific shortcomings. ${ }^{18}$ None has yet been used in India. We developed our own instrument from Indian child health promotion materials, applying a recent systematic review to guide conceptualisation of domains. ${ }^{19}$ Surveyors asked questions in relation to three images ((1) government poster to promote immunisation; (2) poster to promote oral rehydration salts for diarrhoea; (3) India's immunisation card), each of which included pictorial and written elements. Surveyors followed a three-step algorithm:

1. The respondent was invited to identify the topic. No prompts were given. This step tested spontaneous pictorial recognition or literacy.

2. The surveyor then read a short text describing the image and its meaning. Respondents were advised to listen closely as questions based on the passage would follow. The text was read slowly and repeated twice. This was done to provide a level playing field for non-literate respondents.

3. The surveyor posed two factual questions based on the text. After each question, the respondent was invited to reply. Answers were scored as 'correct', 'incorrect' or 'no response' according to a pre-established coding sheet.

The six responses from step 3 were used to categorise participants into site-specific tertiles of high, average or low health literacy via exploratory factor analysis. The tool is available at: https:/www.webdepot.umontreal.ca/Usagers/johrim/ MonDepotPublic/HealthLiteracyTool/HealthLiteracyTool.pdf.
Potential confounders and effect modifiers

\section{Sociodemographic characteristics}

Maternal and paternal education, maternal age, religion and birth order of child are associated with immunisation in India ${ }^{20}$ and worldwide. ${ }^{21}$ Attitudes towards child sex may be correlated with education. These variables were considered potential confounders. Education was coded into four categories. We also summed maternal and paternal educational categories to create a new seven-category variable ranging from 0 to 6 . We performed principal components analysis to construct a relative index of household wealth from a list of assets developed from India's major national surveys, ${ }^{14} 1522$ and used this index to divide the sample into quintiles. ${ }^{23}$

\section{Service delivery}

In India, the lowest level of immunisation delivery takes place at the village or slum cluster level. Government norms require that health workers give a verbal reminder to eligible households before each immunisation day. ${ }^{24}$ We created four variables to study service delivery variations. These were, the proportion of households per village who reported: (1) never having received an immunisation reminder; (2) a late immunisation reminder; (3) poor service quality as a reason for their child's incomplete vaccination; (4) lack of access as a reason for their child's incomplete vaccination.

\section{Measures to address potential biases}

(1) Our principal challenge was to develop an instrument to measure health literacy that was conceptually well motivated, understood by our target population, able to be asked and scored in a standardised way by surveyors, and produced a valid classification. Prototypes were developed in 2011 and underwent 17 months of iterative field testing and refinement in populations similar to those for this study. (2) Interviewers and respondents were unaware of the principal study hypothesis. They were informed that our goal was to understand how mother's knowledge affects child health. The questionnaire encompassed immunisation, diarrhoea, nutrition and water quality (see web appendix: study procedures).

\section{Study size}

We used Monte Carlo simulations informed by pilot data to calculate sample size for each site independently. Under specific assumptions, for a significance level of $\alpha=0.05$ and a power of $80 \%, 50$ villages with 20 households each were required in the rural site and 590 households in the urban site. Sample size estimates were inflated to account for missing data (see web appendix: study size).

\section{Statistical methods}

For each site, we used factor analysis to allocate participants into health literacy tertiles. ${ }^{25}$ Descriptive analyses used standard techniques (see web appendix: creation of a health literacy variable).

\section{Main analyses}

We used multivariate logistic regression to investigate the relationship between maternal health literacy and vaccination status. Analysis approaches were prespecified and performed separately for rural and urban sites. For the rural site, we analysed data using a multilevel logistic regression model with children, mothers and households (level 1) nested in villages (level 2). In the urban site, we used logistic regression with dummy variables 
to represent measured and unmeasured sources of cluster-level variation. Sociodemographic variables considered as potential confounders and effect modifiers were prespecified based on the scientific literature and used in rural and urban analyses. For the rural site, candidate variables to explain variations in villagelevel service delivery were identified through the scientific literature and refined empirically. Full modelling strategies are described in the web appendix: statistical methods.

\section{Other analyses}

For both sites, we evaluated the relationship across subgroups defined by maternal education and explored a potential interaction between maternal health literacy and mother's education. Analyses for subgroups and interactions were empirically motivated.

We also performed sensitivity analyses. We studied maternal education rather than parental education score. In the rural site, we considered random slopes for each of the level 2 variables. To assess fixed versus random effects modelling choices, we ran an alternative fixed effects model including all level 1 variables from model 3 and 59 dummy variables for villages. We evaluated treatment heterogeneity through random slopes models. In the urban site, to assess the implications of fixed versus random effects assumptions, we ran alternative models representing cluster as a random effect. We assessed whether the effect of health literacy on vaccination uptake differed significantly between urban and rural settings by estimating a combined mixed model with 69 clustering variables (60 villages and 9 slum clusters). Models included an indicator variable for setting (urban $=1$, rural $=0$ ) and interaction terms between the setting indicator and the health literacy variables.

Analyses were restricted to individuals with complete data on all variables. Associations with a $\mathrm{p}$ value of less than 0.05 were viewed as significant. Analyses were implemented in Stata V.13.

\section{RESULTS}

\section{Participants}

In the rural site, 1192 women from 60 villages were confirmed eligible and included in the study; 98.2\% (1170/1192) were included in the analysis. In the urban site, 685 women from nine clusters were confirmed eligible and included in the study; $97.8 \%(670 / 685)$ were included in the analysis.

\section{Descriptive and outcome data}

Table 1 presents descriptive and outcome $e^{15}$ data for the analysis sample (web appendix table S2 provides the complete sample). Study populations were highly deprived; indicators show an urban advantage. DTP3 coverage was $41.9 \%(490 / 1170)$ in the rural site and $80.5 \%(539 / 670)$ in the urban site. The most recent Government of India estimates for DTP3 coverage were $54.4 \%{ }^{15}$ in Hardoi district and $79.0 \%$ in Delhi ${ }^{16}$ (web appendix table S1 provides comparative data). Associations between DTP3 vaccination and education, religion, child sex, birth order and economic status resemble those previously reported in India. ${ }^{20}$ Health literacy was associated with DTP3 vaccination in rural (low health literacy 33.5\% (156/490) DTP3; high health literacy 49.1\% (192/490) DTP3) and urban (low health literacy 74.8\% (214/539) DTP3; high health literacy 88.7\% (197/539) DTP3) sites. Health literacy varied within categories of maternal education (table 2) and parental education (see web appendix table S3). The most important reason given by mothers for their child's incomplete immunisation was lack of awareness of vaccines or the vaccination schedule $(35.9 \%$ (306/852) rural; 20.4\% (39/191) urban; see web appendix table S4). Service delivery problems were more frequent in the rural $(30.0 \%$ $(256 / 852))$ than the urban $(1.5 \%(10 / 685))$ area (see web appendix table S4).

\section{Main results}

A positive association between health literacy and DTP3 vaccination was found in crude and adjusted models for each site (figure 1). In the rural area, model R1 describing the crude association showed a higher likelihood of DTP3 vaccination among mothers with medium $(\mathrm{OR}=1.74,95 \% \mathrm{CI} 1.25$ to 2.42 , $\mathrm{p}=0.001)$ or high $(\mathrm{OR}=1.88,95 \%$ CI 1.38 to $2.57, \mathrm{p}<0.001)$ health literacy, as compared with mothers with low health literacy. After adjustment for maternal and paternal education (model R2), the association between health literacy and DTP3 vaccination was somewhat attenuated for those with medium health literacy $(\mathrm{OR}=1.64,95 \% \mathrm{CI} 1.17$ to $2.30, \mathrm{p}=0.004)$ and no longer significant for those with high health literacy $(\mathrm{OR}=1.38,95 \%$ CI 0.96 to $1.98, \mathrm{p}<0.081)$. Adjustment for all sociodemographic confounders (model R3) yielded little further change $(\mathrm{OR}=1.62,95 \% \mathrm{CI} 1.15$ to $2.30, \mathrm{p}=0.006$ for medium health literacy, and $\mathrm{OR}=1.38,95 \%$ CI 0.94 to $2.03, \mathrm{p}=0.099$ for high health literacy). Fully adjusted model R4 including additional village-level service delivery characteristics resulted in slight further attenuation (table 3). Web appendix table S5 gives random effects for all rural models.

In the urban area, the crude association (model U1) between health literacy and DTP3 vaccination was $\mathrm{OR}=1.36(95 \% \mathrm{CI}$ 0.84 to $2.19, \mathrm{p}=0.212$ ) for mothers with medium health literacy and $\mathrm{OR}=2.70$ (95\% CI 1.63 to $4.47, \mathrm{p}<0.001)$ for mothers with high health literacy, as compared with mothers with low health literacy. After adjustment for maternal and paternal education (model U2), the association between health literacy and DTP3 vaccination was attenuated but remained significant for those with high health literacy (medium health literacy $\mathrm{OR}=1.11,95 \%$ CI 0.66 to $1.85, \mathrm{p}=0.698$; high health literacy $\mathrm{OR}=1.9895 \%$ CI 1.03 to $3.80, \mathrm{p}<0.040$ ). Adjustment for all sociodemographic confounders (model U3) produced little further change (table 3).

\section{Other analyses}

Confounder-adjusted estimates derived under alternative fixed and random effects assumptions were very similar (table 4). Analyses using maternal education as a potential confounder were similar to results using parental education score (see web appendix tables S6 and S7). We found no evidence of an interaction between maternal education and health literacy, or of treatment heterogeneity. We found no differences between the effects of health literacy in rural and urban settings (see web appendix table S8).

\section{DISCUSSION}

\section{Principal findings}

We hypothesised that maternal health literacy is positively associated with children's receipt of vaccines after adjustment for confounding. This hypothesis was confirmed in each of our study sites. The crude relationship between health literacy and DTP3 completion was significant in urban and rural settings for medium and high health literacy categories. In the rural site after adjustment for maternal (age and education), paternal (education), child (sex, birth order), household (religion, wealth quintile) and service delivery (access, quality, receipt of reminders) characteristics, medium health literacy was associated with greater DTP3 completion as compared with low health literacy, while high health literacy was not associated with the outcome. 
Table 1 Characteristics of rural and urban study samples, India 2013 (analysis sample)

\begin{tabular}{|c|c|c|c|c|}
\hline \multirow[b]{2}{*}{ Characteristics of mothers, children and households } & \multicolumn{2}{|c|}{ Hardoi, Uttar Pradesh* (rural, N=1170) } & \multicolumn{2}{|c|}{ Kirti Nagar, New Delhit (urban, $N=670$ ) } \\
\hline & Total N (\%) & n (\%) of DTP3‡ & Total N (\%) & n (\%) of DTP3‡ \\
\hline Total & $1170(100.0)$ & $490(41.9)$ & $670(100.0)$ & $539(80.5)$ \\
\hline \multicolumn{5}{|l|}{ Health literacy } \\
\hline Low & $466(39.8)$ & $156(33.5)$ & $286(42.7)$ & $214(74.8)$ \\
\hline Medium & $313(26.8)$ & $142(45.4)$ & $162(24.2)$ & $128(79.0)$ \\
\hline High & $391(33.4)$ & $192(49.1)$ & $222(33.1)$ & $197(88.7)$ \\
\hline \multicolumn{5}{|l|}{ Maternal education } \\
\hline None $(0)$ & $680(58.1)$ & $253(37.2)$ & $303(45.2)$ & $229(75.6)$ \\
\hline Some primary (grades $1-5$ ) & $84(7.2)$ & $37(44.1)$ & $125(18.7)$ & $99(79.2)$ \\
\hline Some upper primary (grades $6-8$ ) & $215(18.4)$ & $93(43.3)$ & $111(16.6)$ & $95(85.6)$ \\
\hline Some secondary or higher ( $\geq$ grade 9 ) & $191(16.3)$ & $107(56.0)$ & $131(19.6)$ & $116(88.5)$ \\
\hline \multicolumn{5}{|l|}{ Paternal education } \\
\hline None $(0)$ & $338(28.9)$ & $115(34.0)$ & $177(26.4)$ & $132(74.6)$ \\
\hline Some primary (grades $1-5$ ) & $203(17.4)$ & $97(47.8)$ & $115(17.2)$ & $90(78.3)$ \\
\hline Some upper primary (grades $6-8$ ) & $202(17.3)$ & $85(42.1)$ & $149(22.2)$ & $124(83.2)$ \\
\hline Some secondary or higher ( $\geq$ grade 9 ) & $427(36.5)$ & $193(45.2)$ & $229(34.2)$ & $193(84.3)$ \\
\hline Mother's age (mean, SD) & $(27.8,5.6)$ & $(27.6,5.3)$ & $(25.2,4.0)$ & $(25.1,4.0)$ \\
\hline \multicolumn{5}{|l|}{ Birth order } \\
\hline 1 & $300(25.6)$ & $150(50.0)$ & $236(35.2)$ & $199(84.3)$ \\
\hline 2 & $260(22.2)$ & $97(37.3)$ & $193(28.8)$ & $151(78.2)$ \\
\hline 3 & $226(19.3)$ & $96(42.5)$ & $140(20.9)$ & $106(75.7)$ \\
\hline 4 & $172(14.7)$ & $72(41.9)$ & $62(9.3)$ & $56(90.3)$ \\
\hline$\geq 5$ & $212(18.1)$ & $75(35.4)$ & $39(5.8)$ & $27(69.2)$ \\
\hline \multicolumn{5}{|l|}{ Child sex } \\
\hline Male & $598(51.1)$ & $262(43.8)$ & $346(51.6)$ & $271(78.3)$ \\
\hline Female & $572(48.9)$ & $228(39.9)$ & $324(48.4)$ & $268(82.7)$ \\
\hline \multicolumn{5}{|l|}{ Religion } \\
\hline Hindu & $1065(91.0)$ & $455(42.7)$ & $605(90.3)$ & $491(81.2)$ \\
\hline Muslim & $105(9.0)$ & $35(33.3)$ & $65(9.7)$ & $48(73.9)$ \\
\hline \multicolumn{5}{|l|}{ Quintile of Wealth Index } \\
\hline 1st quintile (poorest $20 \%$ ) & $275(23.5)$ & $105(38.2)$ & $138(20.6)$ & $105(76.1)$ \\
\hline 2nd quintile & $204(17.4)$ & $78(38.2)$ & $166(24.8)$ & $131(78.9)$ \\
\hline 3rd quintile & $227(19.4)$ & $80(35.2)$ & $107(16.0)$ & $83(77.6)$ \\
\hline 4th quintile & $229(19.6)$ & $107(46.7)$ & $124(18.5)$ & $103(83.1)$ \\
\hline 5th quintile (richest $20 \%$ ) & $235(20.1)$ & $120(51.1)$ & $135(20.2)$ & $117(86.7)$ \\
\hline \multicolumn{5}{|l|}{ Immunisation reminder given late (>1 month ago)§ } \\
\hline No & $644(54.2)$ & $278(43.9)$ & $523(78.1)$ & $411(78.6)$ \\
\hline Yes & $536(45.8)$ & $212(39.5)$ & $147(21.9)$ & $128(87.1)$ \\
\hline \multicolumn{5}{|l|}{ Immunisation reminder never given } \\
\hline No & $1019(87.1)$ & $464(45.5)$ & $616(91.9)$ & 509 (82.6) \\
\hline Yes & $151(12.9)$ & $26(17.2)$ & $54(8.1)$ & $30(55.6)$ \\
\hline
\end{tabular}

\begin{tabular}{|c|c|c|c|c|}
\hline \multirow[b]{2}{*}{ Village/neighbourhood characteristics } & \multicolumn{2}{|c|}{$\begin{array}{l}\text { Hardoi, Uttar Pradesh } \\
\text { (rural, } \mathrm{N}=60 \text { ) }\end{array}$} & \multicolumn{2}{|c|}{$\begin{array}{l}\text { Kirti Nagar, New Delhi } \\
\text { (urban, } \mathrm{N}=9 \text { ) }\end{array}$} \\
\hline & Number & Per cent & Number & Per cent \\
\hline \multicolumn{5}{|l|}{ Village electrification } \\
\hline Not electrified & 8 & 13.3 & 0 & 0.0 \\
\hline$<6 \mathrm{~h}$ & 30 & 50.0 & 3 & 33.3 \\
\hline$\geq 6 \mathrm{~h}$ & 22 & 36.7 & 6 & 66.7 \\
\hline \multicolumn{5}{|l|}{ Any health facility in village/neighbourhood } \\
\hline Yes & 58 & 96.7 & 9 & 100.0 \\
\hline No & 2 & 3.3 & 0 & 0.0 \\
\hline Service delivery problems & Mean (SD) & Range & Mean (SD) & Range \\
\hline Proportion of parents citing lack of access as reason for child's incomplete immunisation & $14.5(19.8)$ & $0.0-0.95$ & $2.4(3.4)$ & $0.0-0.13$ \\
\hline Proportion of parents citing poor service quality as reason for child's incomplete immunisation & $9.2(16.0)$ & $0.0-0.95$ & $2.4(3.3)$ & $0.0-0.09$ \\
\hline Proportion of parents who received a late reminder & $45.8(20.2)$ & $0.05-0.95$ & $21.9(21.2)$ & $0.0-0.61$ \\
\hline Proportion of parents who never received a reminder & $13.0(15.6)$ & $0.0-0.75$ & $8.1(8.9)$ & $0.0-0.43$ \\
\hline \multicolumn{5}{|c|}{$\begin{array}{l}\text { *For the rural site, } 1.9 \% \text { ( } 22 \text { of } 1192) \text { respondents had missing values and were dropped from the analysis sample. The following values were missing by variable: birth order } 0.8 \% \\
(10 / 1192) \text {; religion } 0.5 \%(6 / 1192) \text {; reminder last year } 0.5 \%(6 / 1192) \text {; reminder never } 0.5 \%(6 / 1192) \text { and health literacy } 0.2 \%(2 / 1192) . \\
\text { FFor the urban site, } 2.2 \%(15 \text { of } 685) \text { respondents had missing values and were dropped from the analysis sample. The following values were missing by variable: birth order } 1.6 \% \\
(11 / 685) \text {; reminder last year } 0.3 \%(2 / 685) \text {; reminder never } 0.3 \%(2 / 685) \text {; health literacy } 0.1 \%(1 / 685) \text { and mother's age } 0.1 \%(1 / 685) \text {. } \\
\text { ‡Column refers to percentage of participants receiving three doses of diphtheria-tetanus-pertussis vaccine 'DTP3'. } \\
\text { §We asked each mother "When did someone last come to your home to give information about immunisation?" Those who replied "within the last month" or "before the last } \\
\text { immunisation day" were considered to have received an 'on-time' reminder; else, the reminder was considered 'late'. }\end{array}$} \\
\hline
\end{tabular}


Table 2 Relationship between health literacy and maternal education in rural and urban sites, India 2013

\begin{tabular}{|c|c|c|c|c|c|c|}
\hline \multirow[b]{2}{*}{ Health literacy } & \multicolumn{3}{|c|}{ Hardoi, Uttar Pradesh (rural, N=1170) } & \multicolumn{3}{|c|}{ Kirti Nagar, New Delhi (urban, N=670) } \\
\hline & Low $n(\%)$ & Medium n (\%) & High n (\%) & Low $n(\%)$ & Medium n (\%) & High n (\%) \\
\hline \multicolumn{7}{|l|}{ Maternal education } \\
\hline None (0) & $396(58.2)$ & $204(30.0)$ & $80(11.8)$ & $235(77.5)$ & $59(19.5)$ & $9(3.00)$ \\
\hline Some primary (grades 1-5) & $13(15.5)$ & $10(11.9)$ & $61(72.6)$ & $37(29.6)$ & $49(39.2)$ & $39(31.2)$ \\
\hline Some upper primary (grades 6-8) & $43(20.0)$ & $68(31.6)$ & $104(48.4)$ & $8(7.2)$ & $36(32.4)$ & $67(60.4)$ \\
\hline Some secondary or higher ( $\geq$ grade 9 ) & $14(7.3)$ & $31(16.2)$ & $146(76.4)$ & $6(4.6)$ & $18(13.7)$ & $107(81.7)$ \\
\hline
\end{tabular}

In the urban site after adjustment for maternal (age and education), paternal (education), child (sex, birth order), household (religion, wealth quintile) and slum cluster characteristics, high health literacy benefitted DTP3 completion, while medium health literacy conferred no advantage over low health literacy.
Health services are a critical determinant of immunisation coverage, and villages or clusters are India's service delivery points closest to communities. The main analysis for the rural site used a random effects model accounting for village-level service delivery performance, while the main urban analysis
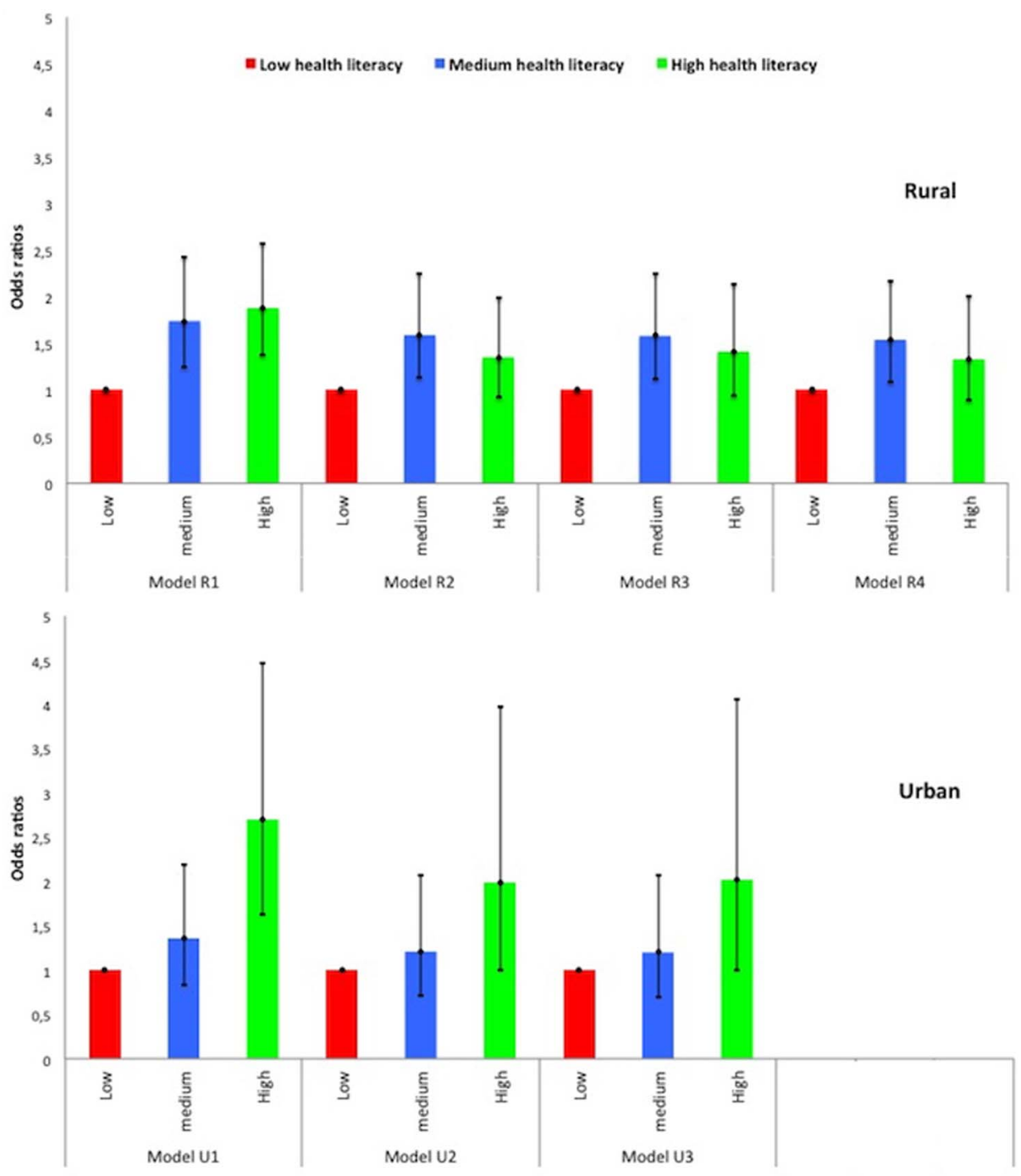

Created by Paint $X$

Figure 1 Crude and adjusted association between maternal health literacy and receipt of three doses of diphtheria-tetanus-pertussis (DTP3) vaccine among children 12-23 months, India 2013. Statistical models for the rural site: model R1—crude association between maternal health literacy and child's DTP3 status; model R2 - model R1 adjusted for parental education score; model R3 - model R2 adjusted for maternal (age), child (sex, birth order) and household (religion, wealth quintile) characteristics; model R4-model R3 adjusted for village-level service delivery (access, quality, receipt of reminders). Statistical models for the urban site: model U1_crude association between maternal health literacy and child's DTP3 status; model U2-model U1 adjusted for parental education score; model U3-model U2 adjusted for maternal (age), child (sex, birth order) and household (religion, wealth quintile) characteristics. 
Table 3 Fully adjusted models of the association between maternal health literacy and receipt of DTP3 vaccine among children 12-23 months, India 2013

\begin{tabular}{|c|c|c|c|c|c|c|}
\hline & \multicolumn{3}{|c|}{$\begin{array}{l}\text { Hardoi, Uttar Pradesh (rural, } \\
\mathrm{N}=1170 \text { ) model R4 }\end{array}$} & \multicolumn{3}{|c|}{$\begin{array}{l}\text { Kirti Nagar, New Delhi (urban, } \\
\mathrm{N}=670 \text { ) model U3 }\end{array}$} \\
\hline & $\mathrm{OR}$ & $(95 \% \mathrm{Cl})$ & $\mathrm{p}$ Value & $\mathrm{OR}$ & $(95 \%$ Cl) & $\mathrm{p}$ Value \\
\hline \multicolumn{7}{|l|}{ Health literacy } \\
\hline Low & Reference & & & Reference & & \\
\hline Medium & 1.57 & (1.11 to 2.20 ) & 0.015 & 1.11 & (0.65 to 1.88 ) & 0.705 \\
\hline High & 1.30 & (0.89 to 1.91 ) & 0.175 & 2.06 & (1.06 to 3.99 ) & 0.032 \\
\hline \multicolumn{7}{|l|}{ Parental education score } \\
\hline 0 & Reference & & & Reference & & \\
\hline 1 & 1.62 & (0.94 to 2.80 ) & 0.082 & 0.90 & (0.46 to 1.75$)$ & 0.750 \\
\hline 2 & 1.11 & (0.72 to 1.72 ) & 0.638 & 1.26 & (0.64 to 2.48 ) & 0.507 \\
\hline 3 & 1.51 & (1.00 to 2.29 ) & 0.048 & 1.20 & (0.62 to 2.31 ) & 0.592 \\
\hline 4 & 2.22 & (1.29 to 3.81 ) & 0.004 & 2.16 & (0.91 to 5.15$)$ & 0.081 \\
\hline 5 & 1.24 & (0.72 to 2.16$)$ & 0.435 & 1.43 & (0.55 to 3.69 ) & 0.464 \\
\hline 6 & 2.64 & (1.46 to 4.78 ) & 0.001 & 1.30 & (0.48 to 3.53$)$ & 0.598 \\
\hline Mother 's age & 1.02 & (0.99 to 1.06 ) & 0.195 & 1.00 & (0.94 to 1.07 ) & 0.879 \\
\hline \multicolumn{7}{|l|}{ Birth order of child } \\
\hline 1 & Reference & & & & & \\
\hline 2 & 0.55 & (0.37 to 0.82 ) & 0.003 & 0.70 & (0.41 to 1.20$)$ & 0.194 \\
\hline 3 & 0.62 & (0.40 to 0.96$)$ & 0.033 & 0.72 & (0.38 to 1.34$)$ & 0.298 \\
\hline 4 & 0.72 & (0.43 to 1.20$)$ & 0.210 & 2.35 & (0.83 to 6.67$)$ & 0.110 \\
\hline$\geq 5$ & 0.58 & (0.33 to 1.02 ) & 0.064 & 0.57 & (0.21 to 1.60$)$ & 0.288 \\
\hline \multicolumn{7}{|l|}{ Child sex } \\
\hline Female (reference male) & 0.83 & (0.63 to 1.08$)$ & 0.157 & 1.36 & (0.90 to 2.05$)$ & 0.147 \\
\hline \multicolumn{7}{|l|}{ Religion } \\
\hline Muslim (reference Hindu) & 0.57 & (0.34 to 0.94$)$ & 0.027 & 0.89 & (0.46 to 1.72$)$ & 0.732 \\
\hline \multicolumn{7}{|l|}{ Quintile of Wealth Index } \\
\hline 1st quintile (poorest $20 \%$ ) & Reference & & & & & \\
\hline 2nd quintile & 1.00 & (0.65 to 1.54$)$ & 0.986 & 1.01 & (0.54 to 1.93$)$ & 0.986 \\
\hline 3rd quintile & 0.82 & (0.54 to 1.25$)$ & 0.359 & 1.00 & (0.48 to 2.06$)$ & 0.997 \\
\hline 4th quintile & 1.10 & (0.72 to 1.68$)$ & 0.656 & 1.20 & (0.58 to 2.51$)$ & 0.626 \\
\hline 5th quintile (richest 20\%) & 1.26 & (0.82 to 1.94$)$ & 0.298 & 1.75 & (0.80 to 3.79$)$ & 0.160 \\
\hline Proportion of parents citing lack of access as reason for child's incomplete immunisation & 0.16 & (0.04 to 0.68$)$ & 0.012 & - & - & - \\
\hline $\begin{array}{l}\text { Proportion of parents citing poor service quality as reason for child's incomplete } \\
\text { immunisation }\end{array}$ & 0.95 & (0.15 to 6.08$)$ & 0.954 & - & - & - \\
\hline Proportion of parents who received a late reminder & 0.35 & (0.11 to 1.13$)$ & 0.079 & - & - & - \\
\hline Proportion of parents who have never received a reminder & 0.01 & (0.00 to 0.11$)$ & 0.000 & - & - & - \\
\hline \multicolumn{7}{|l|}{ Cluster (neighbourhood number) } \\
\hline 1 & Reference & - & - & 1.00 & & \\
\hline 2 & - & - & - & 0.49 & (0.12 to 2.00$)$ & 0.324 \\
\hline 3 & - & - & - & 0.70 & (0.21 to 2.32 ) & 0.558 \\
\hline 4 & - & - & - & 1.44 & (0.35 to 6.03$)$ & 0.615 \\
\hline 5 & - & - & - & 0.21 & (0.04 to 1.07$)$ & 0.060 \\
\hline 6 & - & - & - & 0.80 & (0.23 to 2.74 ) & 0.720 \\
\hline 7 & - & - & - & 0.93 & (0.21 to 4.19$)$ & 0.923 \\
\hline 8 & - & - & - & 0.80 & (0.23 to 2.80$)$ & 0.737 \\
\hline 9 & - & - & - & 1.63 & (0.45 to 5.88$)$ & 0.455 \\
\hline \multicolumn{7}{|c|}{$\begin{array}{l}\text { Parental education score is the sum of maternal and paternal education categories ('0' none; } 1 \text { 'some primary'; ' } 2 \text { ' some upper primary; ' } 3 \text { ' some secondary or higher). It ranges from } \\
\text { '0' both parents have received no schooling to ' } 6 \text { ' both parents have attended secondary or higher. } \\
\text { Model R4-adjusted for parental education score, maternal (age), child (sex, birth order), household (religion, wealth quintile) and village-level service delivery (access, quality, receipt } \\
\text { of reminders) characteristics. } \\
\text { Model U3-adjusted for parental education score, maternal (age), child (sex, birth order) and household (religion, wealth quintile) characteristics. Fixed effects capture variations among } \\
\text { neighbourhoods. } \\
\text { DTP3, three doses of diphtheria-tetanus-pertussis. }\end{array}$} \\
\hline
\end{tabular}

used fixed effects to represent observed and unobserved variation between clusters. In each site, sensitivity analyses comparing fixed versus random effects assumptions yielded virtually identical estimates of the association between health literacy and DTP3 vaccination. The negative finding concerning treatment heterogeneity suggests a similar relationship between health literacy and child vaccination status in each rural village. A sensitivity analysis found no difference between the effect of health literacy in rural and urban settings. The joint distribution of health literacy and parental education scores may differ across settings and may partly explain differences observed between sites. 
Table 4 ORs and 95\% Cls for fully adjusted rural and urban models showing the effect of maternal health literacy on child's proportion of DTP3 coverage estimated under fixed effects and random effects assumptions

\begin{tabular}{|c|c|c|c|c|c|c|}
\hline \multirow[b]{3}{*}{ Variables } & \multicolumn{6}{|c|}{ Hardoi, Uttar Pradesh (rural, N=1170) } \\
\hline & \multicolumn{3}{|c|}{ Random effects (model R4) } & \multicolumn{3}{|c|}{ Fixed effects (model R3FE) } \\
\hline & OR & $(95 \% \mathrm{Cl})$ & p Value & OR & $(95 \% \mathrm{Cl})$ & p Value \\
\hline \multicolumn{7}{|c|}{ Health literacy } \\
\hline Low & \multicolumn{3}{|l|}{ Reference } & \multicolumn{3}{|c|}{ Reference } \\
\hline Medium & 1.57 & (1.11 to 2.21 ) & 0.010 & 1.64 & (1.14 to 2.35 ) & 0.007 \\
\hline \multirow[t]{4}{*}{ High } & 1.30 & (0.89 to 1.91$)$ & 0.172 & 1.34 & $(0.84$ to 2.00$)$ & 0.149 \\
\hline & \multicolumn{6}{|c|}{ Kirti Nagar, New Delhi (urban, N=670) } \\
\hline & \multicolumn{3}{|c|}{ Random effects (model U3RE) } & \multicolumn{3}{|c|}{ Fixed effects (model U3) } \\
\hline & OR & $(95 \% \mathrm{Cl})$ & p Value & OR & $(95 \% \mathrm{Cl})$ & p Value \\
\hline \multicolumn{7}{|c|}{ Health literacy } \\
\hline Low & \multicolumn{3}{|l|}{ Reference } & \multicolumn{3}{|c|}{ Reference } \\
\hline Medium & 1.11 & (0.65 to 1.88$)$ & 0.705 & 1.07 & (0.64 to 1.79 ) & 0.808 \\
\hline High & 2.06 & (1.06 to 3.99$)$ & 0.032 & 2.11 & (1.06 to 4.08$)$ & 0.026 \\
\hline
\end{tabular}

\section{Interpretation}

This study breaks new ground by demonstrating the role of maternal health literacy as a potential determinant of child health in developing countries. Health literacy may be conceived as a personal asset that modifies risk or resilience to disease, ${ }^{26}$ whose unequal distribution contributes to health inequities. Mother's health literacy may be associated with a greater understanding of disease mechanisms, perceived importance of accessing services, motivation to care for her child's health and an improved capacity to negotiate the health system.

This relationship may depend partly on context. Education levels and health systems are particularly weak in the rural site, where even moderate levels of health literacy conferred an advantage. In the urban site, education levels are somewhat higher, health systems stronger, and the effect size associated with high health literacy larger. The finding that health literacy is a determinant of childhood vaccination may be most relevant for LMIC populations in areas with weak to moderate health systems.

Conceptually, the advantage conferred by health literacy is not specific to vaccination. Additional evidence is required to assess its importance for other health conditions. Improved maternal health literacy may have contributed to the beneficial effect of women's groups on maternal and neonatal survival in LMICs. $^{27}$

\section{Strengths and weaknesses}

This study was carefully designed and powered to test the main hypothesis and used appropriate statistical techniques to control for confounding. Study participants are reasonably likely to represent target populations. As compared with findings from external sources, DTP3 coverage rates for the urban sample were virtually identical to Delhi averages. ${ }^{16}$ DTP3 coverage in the rural sample was slightly lower, which could suggest that our survey sample was worse off than the district average, or inaccuracies in the comparison survey for this variable. ${ }^{15}$ Rates of non-participation and missing data were very low. Neither surveyors nor respondents were informed of the study hypothesis. Findings were similar in rural and urban sites with substantially different characteristics and results remained robust through extensive sensitivity analyses.

Results are subject to five important limitations. (1) There is no standard instrument to assess health literacy in this setting. ${ }^{18}$ We developed an instrument that performed well in our target populations, but exposure measurement is likely imprecise. This non-differential bias would diminish the strength of the association. (2) A study of university students in the USA found health literacy as a construct to be closely related to general cognitive ability, ${ }^{6}$ but our survey included no cognitive ability measure. In our study populations, it is likely that structural processes reflecting conceptions of gender and social status over the life course shape both health literacy and apparent cognitive ability, making their relationship difficult to disentangle. This is an area for future research. (3) All LMIC studies on vaccination must reconcile data from two sources, card and maternal recall. We reduced potential for recall bias by taking data on the youngest 12-23 month-old child. We also adapted established local question models ${ }^{14} 1628$ and offered extensive surveyor training and supervision. A recent Indian study found that maternal recall was reasonably accurate and errors non-systematic. ${ }^{28} \mathrm{~A}$ nondifferential bias is likely to diminish the strength of the effect. (4) As data were collected cross-sectionally, we cannot infer a causal relationship between maternal health literacy and vaccination status. (5) Residual confounding remains a possibility.

\section{Relationship to other studies}

This is the first published primary study of maternal health literacy and child health or immunisation in a developing country. A longitudinal cohort study from the USA assessed the relationship between maternal health literacy and early infant immunisation, and found no association. ${ }^{29}$ By contrast, we found that maternal health literacy is distinguishable from formal educational attainment and independently related to child vaccination. Our findings cohere with an extensive literature on maternal education and child health in LMICs that suggest the importance of behavioural pathways leading to greater use of 
health services. ${ }^{3} 30$ These findings also tally well with systematic reviews of the determinants of child vaccination in $\mathrm{LMICs}^{21}$ and in India. ${ }^{20}$ Statistical models consider the complex range of factors affecting child vaccination and confirm the importance of well-known service delivery and sociodemographic characteristics, lending plausibility to the novel health literacy results.

\section{CONCLUSIONS}

A majority of developing countries will not achieve Millennium Development Goal 4 (reduce the mortality rate by two-thirds among children under 5 years) by $2015 .{ }^{31}$ Novel interventions to benefit currently lagging populations are required in the context of intensified efforts post-2015. ${ }^{32}$ Unlike education, health literacy can likely be modified in the short term. This research offers a compelling justification for an intervention study to assess whether initiatives targeting health literacy can improve vaccination coverage and child health outcomes in developing countries.

\section{What is already known on this subject?}

Worldwide, research demonstrates a strong, positive link between parental—particularly maternal-education and child health and survival. Improving population education levels, particularly for women, is an essential but slow child health strategy. Interventions targeting health literacy may yield rapid benefits, but the role of maternal health literacy as a determinant of child health in developing countries is unknown.

\section{What this study adds?}

This is the first primary study to examine the relationship between maternal health literacy and child health or child immunisation in a developing country. After rigorous statistical controls, we found that maternal health literacy was positively associated with children's receipt of vaccines in disadvantaged rural and urban populations in India. Initiatives targeting health literacy should be investigated as possible means to circumvent barriers due to low education and improve vaccination coverage and child health outcomes in developing countries.

\footnotetext{
Author affiliations

${ }^{1}$ Centre de Recherche du Centre Hospitalier de l'Université de Montréal (CRCHUM), Montreal, Québec, Canada

Département d'administration de la santé, École de santé publique, Université de Montréal, Montreal, Canada

${ }^{3}$ Harvard Center for Population and Development Studies, Cambridge,

Massachusetts, USA

${ }^{4}$ Département de médicine sociale et préventive, École de santé publique, Université de Montréal, Montreal, Canada

${ }^{5}$ Pratham Education Foundation (ASER Centre), New Delhi, India

6 |'Université de Daloa, Daloa, Cote d'Ivoire

${ }^{7}$ National Health Systems Resource Centre (NHSRC), Ministry of Health and Family Welfare, Government of India, New Delhi, India
}

Acknowledgements The authors offer sincere thanks to Dr Maria-Victoria Zunzunegui, Université de Montréal; Dr Rukmini Banerji, Dr Wilima Wadhwa and Dr Suman Bhattacharjea (Pratham Education Foundation—ASER Centre); and the remarkable field teams. They also thank two anonymous reviewers for very insightful criticisms and suggestions.
Contributors MJ had principal responsibility for conception and design of the study, statistical analysis, interpretation of data and drafting of the manuscript. SVS contributed to analysis and interpretation of data and revision of the manuscript for important intellectual content. M-PS contributed to conception and design of the study, analysis and interpretation of the data, and revision of the manuscript for important intellectual content. SD contributed to conception and design of the study, data acquisition and revision of the manuscript for important intellectual content. DC contributed to data acquisition and revision of the manuscript for important intellectual content. GKK contributed to statistical analysis and revision of the manuscript for important intellectual content. JKS and SP contributed to conception and design of the study and revision of the manuscript for important intellectual content. All authors approved the final version to be published. All authors agree to be accountable for all aspects of the work in ensuring that questions related to the accuracy or integrity of any part of the work are appropriately investigated and resolved. MJ is the guarantor of the paper.

Funding The Bill \& Melinda Gates Foundation (1067851) and the Canadian Institutes for Health Research (299960) funded this study. The corresponding author had full access to all study data and assumes final responsibility for the decision to submit for publication.

\section{Competing interests None.}

Ethics approval The Pratham Ethics Committee (New Delhi, India) and the Research Ethics Committee of the CHUM (Montréal, Canada, \#12.391).

Provenance and peer review Not commissioned; externally peer reviewed.

Open Access This is an Open Access article distributed in accordance with the Creative Commons Attribution Non Commercial (CC BY-NC 4.0) license, which permits others to distribute, remix, adapt, build upon this work non-commercially, and license their derivative works on different terms, provided the original work is properly cited and the use is non-commercial. See: http://creativecommons.org/ licenses/by-nc/4.0/

\section{REFERENCES}

1 Cleland J. The benefits of educating women. Lancet 2010;376:933-4.

2 Gakidou E, Cowling K, Lozano R, et al. Increased educational attainment and its effect on child mortality in 175 countries between 1970 and 2009: a systematic analysis. Lancet 2010;376:959-74.

3 Cleland JG, Van Ginneken JK. Maternal education and child survival in developing countries: the search for pathways of influence. Soc Sci Med 1988;27:1357-68.

4 Ratzan SC, Parker R. National Library of Medicine current bibliographies in medicine: health literacy (introduction). National Institutes of Health, 2000.

5 Berkman ND, Sheridan SL, Donahue KE, et al. Low health literacy and health outcomes: an updated systematic review. Ann Intern Med 2011;155:97-107.

6 Reeve CL, Basalik D. Is health literacy an example of construct proliferation? A conceptual and empirical evaluation of its redundancy with general cognitive ability. Intelligence 2014;44:93-102.

7 Kickbusch I, Pelikan JM, Apfel F, et al. Health literacy: the solid facts. Copenhagen: WHO Regional Office for Europe, 2013.

8 Office of the Registrar General and Census Commissioner. Provisional population tables: Uttar Pradesh: Census 2011. New Delhi: Government of India, Ministry of Home Affairs, 2012.

9 Ram U, Jha P, Ram F, et al. Neonatal, 1-59 month, and under-5 mortality in 597 Indian districts, 2001 to 2012: estimates from national demographic and mortality surveys. Lancet Global Health 2013;1:e219-26.

10 Government of India Ministry of Panchayati Raj. A note on the backward regions grant fund (BRGF) programme (8th Sept. 2009). New Delhi: National Institute of Rural Development, 2009.

11 Bennett $\mathrm{S}$, Woods T, Liyanage WM, et al. A simplified general method for cluster-sample surveys of health in developing countries. World Health Stat Q 1991;44:98-106.

12 ASER Centre. Annual Status of Education Report (ASER). New Delhi: ASER Centre, 2013.

13 WHO and the United Nations Children's Fund (UNICEF). WHO and UNICEF estimates of national immunization coverage, 2012 revision (completed July 2013).

14 International Institute for Population Sciences (IIPS). District Level Household and Facility Survey (DLHS-3), 2007-8: India. Mumbai: IIPS, 2010.

15 Office of the Registrar General and Census Commissioner. Annual 3 Health Survey: 2010-11. New Delhi: Government of India, Ministry of Home Affairs, 2012.

16 Government of India Ministry of Health and Family Welfare. 2009 Coverage Evaluation Survey. New Delhi: UNICEF India Country Office, 2010.

17 MEASURE DHS. DHS Model Questionnaire-phase 6 (2008-2013) (English, French), 2008.

18 Jordan JE, Osborne RH, Buchbinder R. Critical appraisal of health literacy indices revealed variable underlying constructs, narrow content and psychometric weaknesses. J Clin Epidemiol 2011;64:366-79.

19 Sorensen K, Van den Broucke S, Fullam J, et al. Health literacy and public health: a systematic review and integration of definitions and models. BMC Public Health 2012;12:80. 
20 Mathew JL. Inequity in childhood immunization in India: a systematic review. Indian Pediatr 2012;49:203-23.

21 Rainey JJ, Watkins M, Ryman TK, et al. Reasons related to non-vaccination and under-vaccination of children in low and middle income countries: findings from a systematic review of the published literature, 1999-2009. Vaccine 2011;29:8215-21.

22 International Institute for Population Sciences (IIPS) and Macro International. Final report of the National Family Health Survey (NFHS-3), 2005-06. New Delhi: Government of India, Ministry of Health and Family Welfare, 2007.

23 Filmer D, Pritchett LH. Estimating wealth effects without expenditure data—or tears: an application to educational enrollments in states of India. Demography 2001:38:115-32.

24 India Ministry of Health and Family Welfare. Immunization handbook for health workers. New Delhi: MHFW, 2007:87.

25 Kim J-0, Mueller C. Introduction to factor analysis. What it is and how to do it. Beverly Hills: SAGE, 1978.

26 Nutbeam D. The evolving concept of health literacy. Soc Sci Med 2008; 67:2072-8.
27 Prost A, Colbourn T, Seward N, et al. Women's groups practising participatory learning and action to improve maternal and newborn health in low-resource settings: a systematic review and meta-analysis. Lancet 2013;381: 1736-46.

28 Banerjee AV, Duflo E, Glennerster R, et al. Improving immunisation coverage in rural India: clustered randomised controlled evaluation of immunisation campaigns with and without incentives. BMJ 2010;340:c2220.

29 Pati S, Feemster KA, Mohamad Z, et al. Maternal health literacy and late initiation of immunizations among an inner-city birth cohort. Matern Child Health J 2011;15:386-94.

30 Greenaway ES, Leon J, Baker DP. Understanding the association between maternal education and use of health services in Ghana: exploring the role of health knowledge. J Biosoc Sci 2012;44:733-47.

31 Countdown to 2015: Maternal Newborn \& Child Survival. Fulfilling the health agenda for women and children: the 2014 report. 2014.

32 United Nations. A new global partnership: eradicate poverty and transform economies through sustainable development. The Report of the High-Level Panel of Eminent Persons on the Post-2015 Development Agenda, 2013. 